\title{
Principle and in vivo Application of Measurement of Blood Flow by Heated Thermocouple with Feedback Controlled Heater
}

\author{
Bunji Kondo, * Takashi Soma, ${ }^{*}$ Chikao Uyama, ${ }^{*}$ Tatsuo Sawai, ${ }^{* *}$ \\ Masaru Kobayashi, ${ }^{*}$ Kengo Tsunekawa, ${ }^{\dagger}$ Kikuo Mohri, ${ }^{\dagger}$ \\ Masanao Ikeda, ${ }^{\dagger}$ NaOhiro Ohgushi ${ }^{\dagger}$ and Masugi Sato $^{\dagger}$
}

\begin{abstract}
The heated thermocouple with constant heating current has been used by many authors for the measurement of the regional blood flow.

A new method for the measurement of blood flow is proposed by employing the self-adjusting current heating with a feedback control system. In this method, the temperature difference between the hot and cold junctions is kept almost constant and quite small by setting properly the automatically regulated heating current so that the effects of local heat accumulation by heating current on biological reaction in tissue may be avoided.

Further this method is also superior in linearity, applicability to wider range of flow rate and prompt response than the method of constant current heating on both experimental and theoretical standpoints.
\end{abstract}

$\mathrm{S}^{\mathrm{n}}$ NCE REIN ${ }^{1}$ and GiBBs ${ }^{2}$ initially intended to measure the blood flow using "Thermostromuhr" or heated thermocouple, thermoelectric method has been widely used for the measurement of local blood flow not only in isolated vessels but in such tissues as skin, liver, kidney and spleen.

In spite of the disadvantages of this method such as slow response due to the heat capacity of blood and difficulty of calibration, it has been applied popularly because of simple and flexible electrode and easy embedding of electrode in any tissue without remarkable damage, and continuous and separate recording from closely located spots.

However, as reported ${ }^{3-5}$, the instrument with constant heating current proved to give only qualitative result but rather powerless in quantitative determination ${ }^{6}$. Moreover, the constant heating current irrespectively to any flow rate may result in local heat accumulation or high temperature rise, and such environmental changes together with the introduced changes of vasomoter action ${ }^{7}$ and nerve conduction velocity ${ }^{8}$ may cause the error of in vivo measurement.

The present study has been directed to minimize the biological reaction and keep it constant during the measurement of blood flow by employing the self-adjusting current heating with a feedback control system. The characteristics of constant current (C.C.) heating and self-adjusting current (S.C.) heating methods have been compared in regard to their linearity in response to the variation of the flow rate in model experiments and the manner of response in application to animals.

* Department of Electronics, Kyoto University, Kyoto.

** Department of Electrical Engineering, Kyoto University, Kyoto.

$\dagger$ Department of Surgery, Kyoto University, Kyoto, Japan.

This work was supported by grant of Monbusho-kagaku-kenkyuhi No. 72075 . 


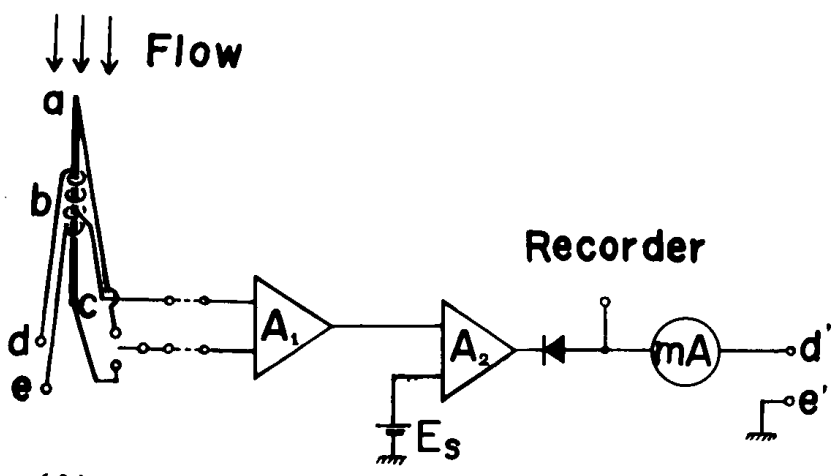

(A)

(B)

Fig. 1. (A) Structure of the electrode and (B) block diagram of the feedback control system. $A_{1}$ and $A_{2}$ : differential amplifiers. $E_{\mathrm{s}}$ : reference voltage to set the temperature difference. a and $c$ : both ends, $b$ : central portion of constant an wire of measuring element. $d$ and $d^{\prime}, e$ and $\mathrm{e}^{\prime}$ are connected respectively during the measurement.

\section{Apparatus and Methods}

The structure of the electrode and the block diagram of the feedback control system are shown in Fig. 1.

The electrode consists of a heater and a measuring element. The measuring element is a constantan wire (about $8 \mathrm{~mm}$ in length and $0.1 \mathrm{~mm}$ in diameter). Electrically insulated copper wires of the same thickness are soldered to both ends ( $a, c$ in Fig. 1) and the center (b in Fig. 1) of the measuring element. Two insulated copper wires are soldered to both ends (d, e in Fig. 1) of another constantan wire (heater : $5 \sim 8 \mathrm{~mm}$ in length and $0.1 \mathrm{~mm}$ in diameter) which is coiled around the central portion of the measuring element.

Constantan wires and their circuits of both elements are perfectly insulated one another only electrically and not thermally ${ }^{9}$.

Considerably small DC current $(50 \mathrm{~mA} \sim 200 \mathrm{~mA}$ ) is initially supplied to the heater so as to keep the temperature difference between the hot and cold junctions by about $0.1^{\circ} \mathrm{C}$.

During the measurement, this temperature difference is maintained as constant as possible by adjusting the heating current automatically following the variation of the flow rate with the feedback control system, and this heating current is recorded as an index of the flow rate. The loop gain of the system should be raised as much as possible within the stable range to reduce the temperature offset.

The variation of temperature difference between the hot and cold junctions is also recorded when the C.C. heating method is applied to this electrode.

For a model experiment of this method, the electrode is designed in a needle form and placed along the central axis of a cylindrical glass tube $(25 \mathrm{~mm}$ in diameter) through which warmed water is perfused. The experiment should be performed with the whole system confined in the constant temperature of $37^{\circ} \mathrm{C}$.

For in vivo experiment of this method, the electrode is inserted into renal cortex of anesthetized dogs and renal blood flow is occluded mechanically.

\section{Principle}

When a point heat source is placed in the unidirectional flow of the homogeneous and isotropic medium, a heat transmission equation is represented as follows :

$$
\frac{\partial \mathrm{F}}{\partial \mathrm{t}}=\frac{\mathrm{k}}{\mathrm{c} \rho} \nabla^{2} \mathrm{~F}-\mathbf{V} \cdot \operatorname{grad} \mathrm{F}+\frac{1}{\mathrm{c}_{\rho} \rho} \mathrm{f}(\mathbf{v}, \mathrm{t})
$$

where $\mathrm{F}$ : temperature of any point in the medium,

$\mathrm{k}$ : thermal conductivity,

$\mathrm{c}$ : specific heat,

$\rho:$ specific weight,

$\mathbf{V}$ : velocity of flow (its absolute value is $\mathrm{V}$ ),

$\mathbf{v}$ : position vector from an origin in the medium,

$f$ : heat generation per unit volume per unit time,

$\mathrm{t}$ : time.

To solve eq. (1), let's postulate the following conditions,

1) heat source is placed on the origin, and supplies a constant (or variable slowly) thermal 


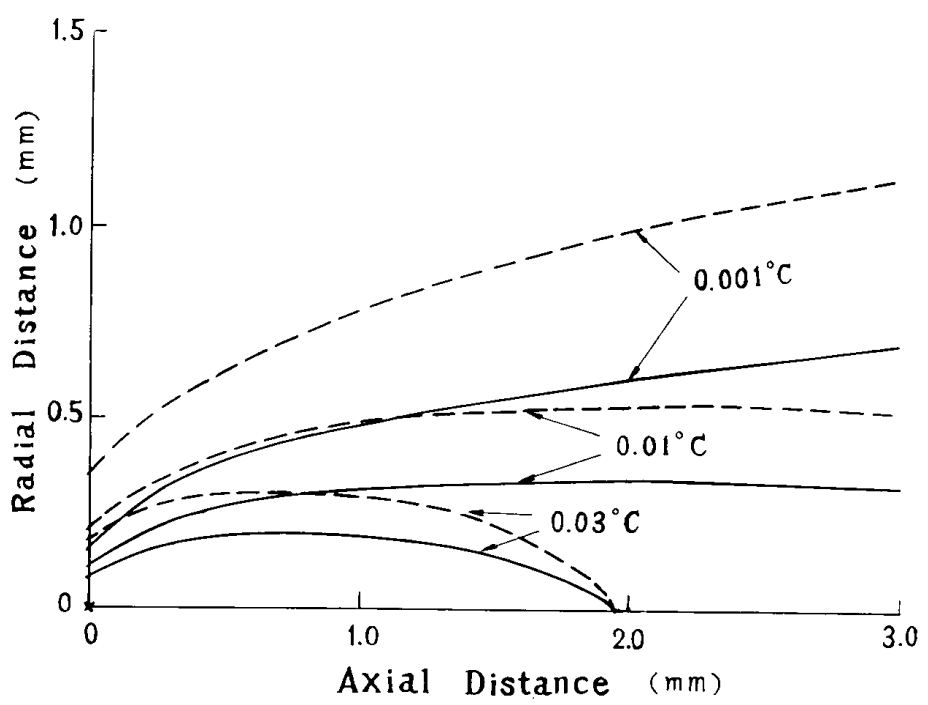

Fig. 2. Steady temperature distribution around a point heater on the origin in the unidirectional water flow. $\mathrm{c}=1, \quad \rho=1, \mathrm{k}=1.4 \times 10^{-3} \mathrm{cal} / \mathrm{cm} \cdot \mathrm{sec} \cdot{ }^{\circ} \mathrm{C}, \mathrm{A}=10^{-4} \mathrm{cal} / \mathrm{sec}$. Solid lines: $\mathrm{V}=1 \mathrm{~cm} / \mathrm{sec}$. Broken lines: $\mathrm{V}=0.4 \mathrm{~cm} / \mathrm{sec}$.

energy A per second,

2) medium spreads uniformly and flows with constant speed.

A coordinate axis $r$ is on the plane perpendicular to the flow direction and another axis $z$ is selected parallel to the flow and its positive direction is to the upstream of the flow in the cylindrical coordinate system. There is no necessity to consider the angular deviation $\phi$ because the system is symmetric with respect to $z$ axis. The solution of eq. (1) is given by

$$
\begin{array}{r}
\mathrm{F}(\mathrm{r}, \mathrm{z}, \mathrm{t})=\frac{\mathrm{A} \cdot \exp \left\{-\mathrm{V}(\mathrm{z}+\mathrm{R}) / 2 \mathrm{a}^{2}\right\}}{8 \pi \mathrm{kR}} \\
\times\left[1+Q\left(\frac{\mathrm{Vt}-\mathrm{R}}{2 \mathrm{a} \cdot \mathrm{t}}\right)\right. \\
\left.+\exp \left(\mathrm{VR} / \mathrm{a}^{2}\right)\left\{1-Q\left(\frac{\mathrm{Vt}+\mathrm{R}}{2 \mathrm{a} / \mathrm{t}}\right)\right\}\right],
\end{array}
$$

where

$$
\begin{aligned}
& \mathrm{a}^{2}=\mathrm{k} / \mu, \quad \mathrm{R}=\left(\mathrm{r}^{2}+\mathrm{z}^{2}\right)^{1 / 2}, \\
& \phi(\mathrm{x})=(2 / v, \bar{\pi}) \int_{\mathrm{o}}^{\mathrm{x}} \exp \left(-\mathrm{u}^{2}\right) \mathrm{du} .
\end{aligned}
$$

To find the steady state solution, we rising tend $t$ to infinity in eq. (2), then obtain

$$
\begin{aligned}
\mathrm{F}(\mathrm{r}, \mathrm{z}, \infty)= & (\mathrm{A} / 4 \pi \mathrm{kR}) \exp \\
& \left\{-\mathrm{V}(\mathrm{R}+\mathrm{z}) / 2 \mathrm{a}^{2}\right\} .
\end{aligned}
$$

Fig. 2 shows a steady temperature distribution when the point heat source is placed in the uniform directional water flow of 1.0 and 0.4 $\mathrm{cm}$ per second. Equithermal lines of $0.01^{\circ} \mathrm{C}$ rise spread at most $0.5 \mathrm{~mm}$ from $z$ axis, besides as the flow speed increases the equithermal lines come close to $z$ axis, therefore the abovementioned assumption 2) is not violated in the measurement of the flow in a comparatively small tube as a blood vessel. To simplify the problem, let's regard the source temperature as the average value within a sphere of radius $\varepsilon$ including a point source as a center. Thus the average temperature is given by

$$
\begin{aligned}
& \mathrm{T}=\int_{-\varepsilon}^{\varepsilon} \mathrm{d} z \int_{0}^{\sqrt{\varepsilon^{2}-z^{2}}} 2 \pi \mathrm{r} \cdot \mathrm{F}(\mathrm{r}, \mathrm{z}, \infty) \\
& \mathrm{dr} /\left(4 \pi \varepsilon^{3} / 3\right) \text {. }
\end{aligned}
$$

Substituting eq. (4) into eq. (5) yields

$$
\begin{aligned}
& \mathrm{T}=\frac{3 \mathrm{a}^{2} \mathrm{~A}}{4 \pi \varepsilon^{2} \mathrm{Vk}} {\left[1-\frac{\mathrm{a}^{2}}{\varepsilon \mathrm{V}}\right.} \\
&\left.\times\left\{1-\exp \left(-\varepsilon \mathrm{V} / \mathrm{a}^{2}\right)\right\}\right],
\end{aligned}
$$

a d if the flow speed tends to zero, the average source temperature becomes

$$
\mathrm{T}_{\mathrm{o}}=3 \mathrm{~A} /(8 \pi \varepsilon \mathrm{k})
$$

now putting

$$
\mathrm{u}=\varepsilon \mathrm{V} / \mathrm{a}^{2}=\mathrm{c} \rho \varepsilon \mathrm{V} / \mathrm{k},
$$

then a flow-temperature relation of the C.C. heating is given by 


$$
\begin{aligned}
\mathrm{y}= & \left(\mathrm{T}_{\mathrm{o}}-\mathrm{T}\right) / \mathrm{T}_{\mathrm{o}} \\
& =\left\{(\mathrm{u}-1)^{2}+1-2 \exp (-\mathrm{u})\right\} / \mathrm{u}^{2} .
\end{aligned}
$$

For sufficiently large $u, y$ is represented approximately by a following rectangular hyperbola

$$
\mathrm{y} \cong 1-2 / \mathrm{u}
$$

and for $0<\mathrm{u}<1$, by a following relation of square root

$$
\mathrm{y} \simeq \mathrm{u} / 3-\mathrm{u}^{2} / 12
$$

Thermal energy $A$ is also represented by means of the heating current $I$ as follows

$$
\mathrm{A}=\mathrm{I}^{2} \mathrm{R} / \mathrm{J} \text {, }
$$

where $\mathrm{J}$ : mechanical equivalent of heat,

$\mathrm{R}$ : electrical resistance of heater wire,

then a flow-current relation of the S.C. heating is given by

$$
\begin{aligned}
\mathrm{x} & =\left(\mathrm{I}-\mathrm{I}_{\mathrm{o}}\right) / \mathrm{I}_{\mathrm{o}} \\
& =\mathrm{u} /[2\{\mathrm{u}-1+\exp (-\mathrm{u})\}]^{1 / 2}-1,
\end{aligned}
$$

where $I_{\mathrm{o}}$ is the heating current necessary to keep prescribed temperature difference when there is no flow.

Thus the relations of eq. (9') and eq. (13) in C.C. and S.C. heating methods respectively, which denote the index of the flow rate, are shown in Fig. 3, where solid lines are obtained by taking $\varepsilon$ as $0.1 \mathrm{~mm}$ and broken lines as 1

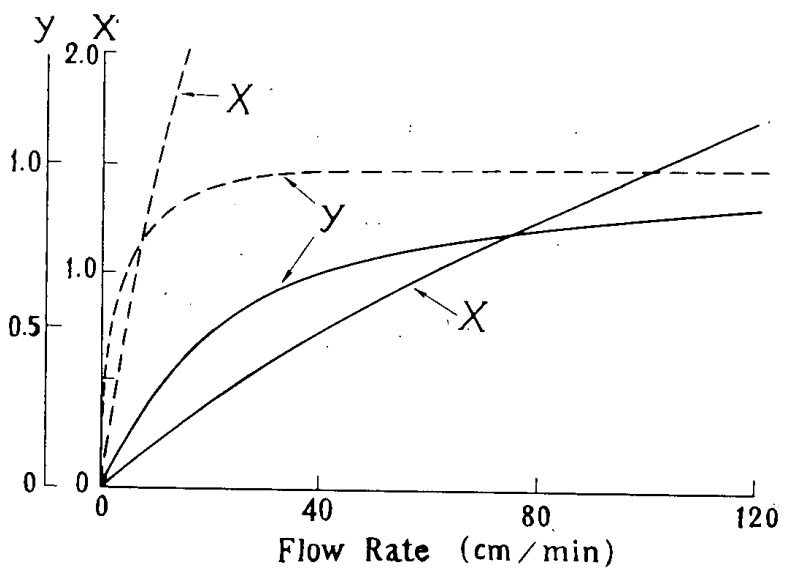

Fig. 3. Theoretical relation between relative decrease of temperature difference $y$ and flow rate in constant current heating method and that between relative increase of heating current $x$ and flow rate in self-adjusting current heating method. $\mathrm{c}=1, \rho=1, \mathrm{k}=1.4 \times 10^{-3} \mathrm{cal} / \mathrm{cm} \cdot \mathrm{sec} \cdot{ }^{\circ} \mathrm{C}$, $\mathrm{A}=10^{-4} \mathrm{cal} / \mathrm{sec}$.

Solid lines: equivalent radius $\varepsilon=0.1 \mathrm{~mm}$. Broken lines : $\varepsilon=1 \mathrm{~mm}$. $\mathrm{mm}$. If the value of equivalent radius $\varepsilon$ is selected properly, the calculated value may coincide quantitatively with the experimental result. It can be easily seen from Fig. 3 that the S.C. heating method is superior in linearity and applicability to wider range of the flow rate than the C.C. heating method, and these facts are in accordance with the following results of the experiments.

\section{RESUlts}

In the model experiment, the temperature difference caused by the variation of the flow in the C.C. heating method was apparently regarded as linear only over the very narrow range of the flow rate, and when the flow rate increased further, the decrease of the decre-

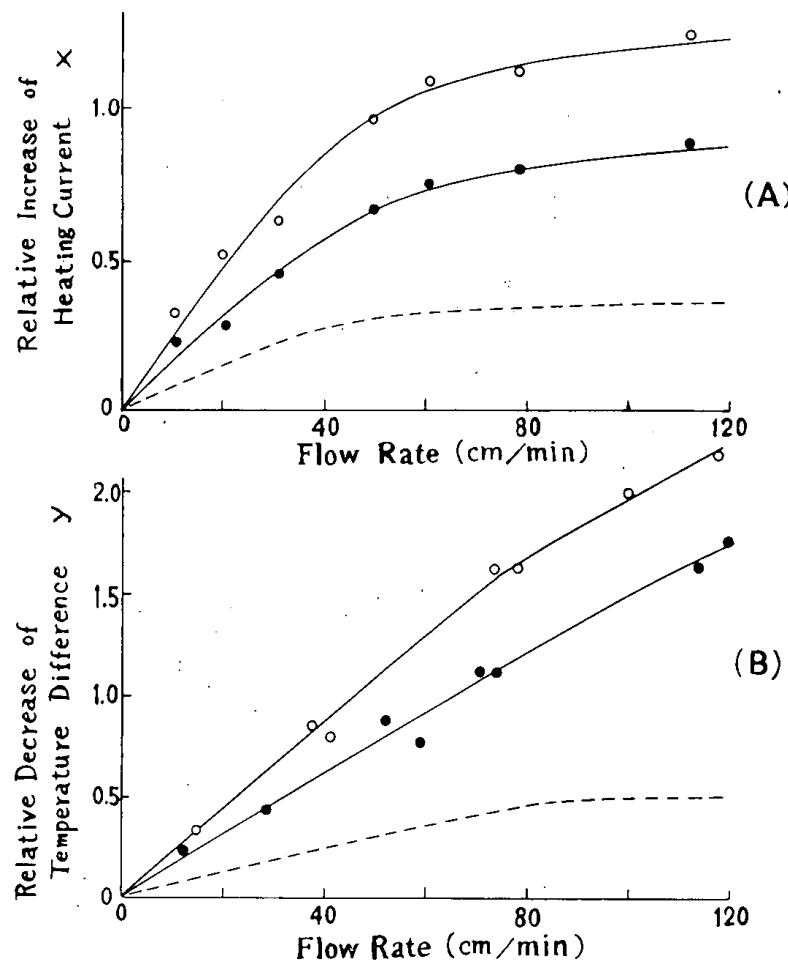

Fig. 4. (A) Relation between relative decrease of temperature difference and flow rate in constant current heating method and (B) relation between relative increase of heating current and flow rate in self-adjusting current heating method in model experiment. Needle-formed electrode is placed along the central axis of cylindrical glass tube $(25 \mathrm{~m} \phi)$ through which warmed water is perfused, and whole system is confined in the constant temperature of $37^{\circ} \mathrm{C} .0: \mathrm{b}-\mathrm{c}$ measurement, $\circ$ : $a-m$ measurement, broken lines show $a-c$ measurement. $a, b$ and $\mathrm{c}$ are explained in Fig. 1. 
ment of temperature difference made difficult to detect the variation of the flow rate as shown in Fig. 4.

While in the S.C. heating method, the linearity between the heating current and the flow rate was observed and the variation of the flow rate could be measured over wider range of the flow rate than in the C.C. heating method. These results might accord with the presumed facts by the above-mentioned principle (Fig. 3). Measurement with the hot junction upstream (b-c measurement) gave higher deviations
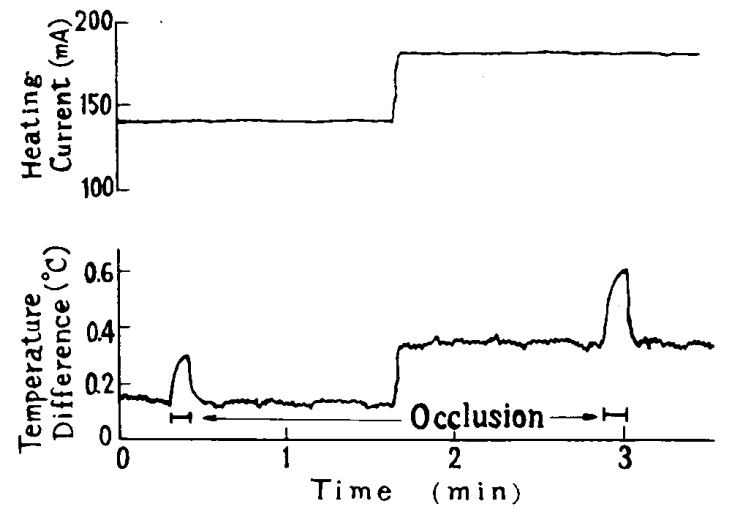

(A)

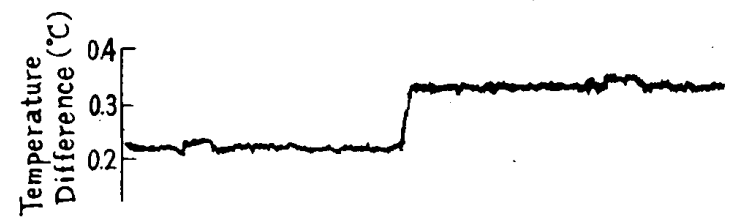

(B)

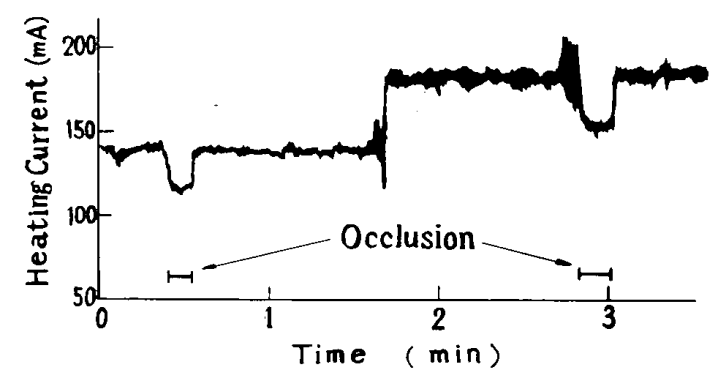

Fig. 5. In vivo experiment applied to renal cortex of an anesthetized dog. The electrode is inserted into left renal cortex and the change in the blood flow is observed when left renal artery is occluded temporarily. Initial heating current is $140 \mathrm{~mA}$ and $180 \mathrm{~mA}$. (A) Change in the flow rate represented as the increase of temperature difference in constant current heating method is $0.14^{\circ} \mathrm{C}$ for $140 \mathrm{~mA}$ heating and $0.22^{\circ} \mathrm{C}$ for $180 \mathrm{~mA}$ heating and (B) change in the flow rate represented as the decrease of heating current in self-adjusting current heating method is $20 \mathrm{~mA}$ for $140 \mathrm{~mA}$ heating and $30 \mathrm{~mA}$ for $180 \mathrm{~mA}$ heating, giving less temperature rise $\left(0.017^{\circ} \mathrm{C}\right.$ and $0.024^{\circ} \mathrm{C}$ respectively) to the tissue examined. than that with the hot junction downstream (a-b measurement), in both methods.

A comparison of the results of in vivo experiment by the C.C. heating and the S.C. heating methods applied to the renal cortex of an anesthetized dog is shown in Fig. 5. When the heating current was $140 \mathrm{~mA}$, the decrease of the temperature difference caused by occulsion of the renal blood flow was $0.14^{\circ} \mathrm{C}$ in the C.C. heating method; on the other hand this difference was $0.017^{\circ} \mathrm{C}$ in the S.C. heating method, and the latter method might give less influence to the tissue examined. Furthermore, the change in the flow rate was represented by a marked drop in the heating current from 140 $\mathrm{mA}$ to $120 \mathrm{~mA}$. Similarly, when the heating current was $180 \mathrm{~mA}$, temporary occlusion of renal blood flow resulted in the decrease of temperature difference of $0.22^{\circ} \mathrm{C}$ in the C.C. heating method and it was $0.024^{\circ} \mathrm{C}$ and the drop in the heating current was $30 \mathrm{~mA}$ in the S.C. heating method. Prompter responses resulted in the S.C. heating method than in the C.C. heating method as shown in Fig. 5.

\section{Discussion}

About the principle of heated thermocouple, J. Grayson ${ }^{10}$ gave a linear relation between the square of heating current $I^{2}$ and the temperature rise $\theta$ according to Carslaw, and showed experimentally this relation in some medium, and derived the apparent thermal conductivity for each medium as a proportional constant. But J. L. LINZELL ${ }^{6}$ pointed out that the square of heating current necessary to elevate the ambient temperature to a prescribed value was not proportional to the flow rate, but rather proportional to square root of it. H. HENSEL et al. ${ }^{11}$ and M. KIESE et al. ${ }^{12}$ also showed the similar results. Recently G. GRÄNGSJö et al. ${ }^{13}$ found the hyperbolic relation between the temperature rise and the perfusion flow, but there was some simplification about the gradient of temperature on the way of the reduction.

To find the principle of heated thermocouple, the authors also postulate some conditions such as unidirectional flow, homogeneous and isotropic medium and regarding the average temperature around the point heat source as the temperature of the heater. 
These approximations will be possible to introduce some deviations between the principle and the experimental results, but a comparison of Fig. 3 and Fig. 4, which are theoretical and experimental results both in the S.C. and C.C. heating methods respectively, shows clearly similar tendencies. And if the value of equivalent radius $\varepsilon$ is selected properly, this principle may explain the experimental results quantitatively as well as qualitatively. Moreover, square root or hyperbolic relation is also obtained as eq. (11) or (10) by proper approximation, and some of the authors used the square root relation to explain the empirical results $^{9}$. About more accurate theoretical or numerical analysis and the optimum frequency characteristic of the system to obtain the most stable or promptest response, the authors have been studying and shall report them in near future. It needs much care of thermal conditions to measure the blood flow by heated thermocouple, but the loop gain of the system should be raised as much as possible within the stable range to reduce the temperature offset.

The structure and size of the electrode also may be carefully decided following the measured object, i.e. whether it is a vein or tissue of an organ or artery. Especially to measure the arterial blood flow, the size of electrodes should be much smaller than above-mentioned dimensions to obtain prompter response.

From the quantitative standpoint, it needs to establish a reliable calibrating method suitable for any organ, but it seems to be very difficult to find it ${ }^{6}$ and SCHER ${ }^{14}$ used a sandfilled glass tube with water. The authors do not fill the sand but satisfactory calibrating methods suitable for various measured objects are not found and under consideration.

It is necessary for clinical use to strengthen the electrode as well as to insulate electrically, then the authors use Ag-araldite compound as the insulater. When DC heating is adopted, the direction of electric current should be determined by taking account of so called "Peltier effect". AC heating method is in progress to avoid Peltier effect.

In the S.C. heating method, the increment of heating current increases as the initial heating current increases, but the latter should be kept to the smallest value in order to obtain better linearity as well as to minimize biological response, except in case of very fast flow.

The reason that the deflection of $b-c$ measurement is greater than that of $a-b$ measurement as is seen in Fig. 4 showing the results of a model experiment, is due to the difference of heat conveyance by flow. The result of $a-c$ measurement is equal to the difference of $b-c$ and $a-b$ measurements, and the direction of the flow can be decided from the polarity of a-c measurement, then if the measuring system has fully quick response to the change of the flow, it may be possible to find the pulsating flow as in an artery.

The S.C. heating method presents prompter response to the change of the flow rate and better linearity as compared with the C.C. heating method of in vivo experiment as shown in Fig. 5. It is perhaps by reason of the heat capacity necessary to change the temperature.

\section{Acknowledgement}

The authors wish to express their sincere gratitude to Prof. Chuji Kimura for his kind advice during this study.

\section{REFERENCE}

1. Rein, H.: Z. Biol. $87: 394$ (1928).

2. Gibbs, F.A.: Proc. Soc. exp. Biol. Med. 31: 141 (1933).

3. Hensel, H., Ruef, J. und Golenhofen, K.: $Z$. Kleisl. forsch. 43 : 756 (1954).

4. Grayson, J. and Johnson, D.H.: J. Physiol. 120 : 73 (1953).

5. Hagihara, Y. and Takahashi, I.: J. Jap. Coll. Angiol. $8: 125$ (1967). (in Japanese)

6. Linzell, J.L.: J. Physiol. $121: 390$ (1953).

7. Abramson, D.I., Chu, L.S.W., Lee, S.W. and Tuck, S. : J. Vascul. Dis. $4: 205$ (1967).

8. Abramson, D.I., Chu, L. S. W., Tuck, S. Jr., Lee, S.W., Richardson, G. And Levin, M. : JAMA 198 : 1082 (1966).

9. Tsunekawa, K., Mohri, K., Ikeda, M., Ohgushi, M., Soma, T. and SawaI, T.: Experientia (to be published).

10. Grayson, J.: J. Physiol. 118 : 54 (1952).

11. Hensel, H., Ruef, J. und Golenhofen, K. : Pfiigers Arch. 259 : 267 (1954).

12. Kiese, M. Und Lange, G.: Arch. exper. Path. u. Pharmakol. 231 : 149 (1957).

13. Grängsjö, G., Sandblom, J., Ulfendahl, H.R. AND Wolgast, M.: Acta physiol. scand. 66: 366 (1966).

14. Scher, A.M.: Am.J. Physiol. $167: 539$ (1951). 